\title{
Antiarrhythmic drug treatment: need for continuous vigilance
}

\author{
Hein JJ Wellens, Joep L Smeets, Marc Vos, Anton P Gorgels
}

\begin{abstract}
Any physician treating cardiac arrhythmias should be aware of a possible worsening of the arrhythmia (so-called arrhythmogenesis) after the start of antiarrhythmic drug treatment. To facilitate the recognition of that complication the type and possible mechanisms of arrhythmogenesis are reviewed. Recognition also requires an understanding of (a) the time of appearance of arrhythmogenic effects after different drugs, (b) the value of non-invasive and invasive tests, and (c) the profile of the patient most likely to develop this problem. Guidelines are given to reduce the incidence of the arrhythmogenic effects of antiarrhythmic drugs and a plea is made that the physician should be always on the alert for this complication.
\end{abstract}

Dr Dennis Krikler's expertise in the electrocardiography and electrophysiology of cardiac arrhythmias is well known and admired worldwide. For his festschrift we selected a topic that has interested him for a long time ${ }^{12}$ : the occurrence of cardiac arrhythmias induced by medication prescribed to treat the arrhythmia.

Many thousands of patients are taking medication because of a cardiac arrhythmia. There is increasing awareness that approximately $15 \%$ of patients show worsening of their arrhythmia or the appearance of a new arrhythmia after they have started antiarrhythmic drug treatment. This so-called arrhythmogenic effect or arrhythmogenesis can be life-threatening. ${ }^{3-7}$

The results of the CAST study ${ }^{89}$ also made the public aware of the possible dangers of antiarrhythmic drug treatment. Physicians treating cardiac arrhythmias need to be developing this problem, and offer guidelines to reduce its incidence.

\section{Types and possible mechanisms of arrhythmogenesis}

It is useful to classify the different types of arrhythmogenic effects ${ }^{5-7}$ (table 1) because it facilitates their recognition and may help to identify possible mechanisms.

\section{WORSENING OF AN ALREADY MANIFEST} ARRHYTHMIA

After antiarrhythmic drug administration the same arrhythmia may occur more frequently, last longer or become less well tolerated because the rate increases. Table 2 shows that this category includes patients with isolated ventricular extrasystoles or non-sustained ventricular tachycardia in whom sustained (fig 1) or even incessant ventricular tachycardia subsequently develops. Patients with atrial flutter and atrial fibrillation in whom druginduced slowing of the atrial rate results in more atrial impulses being conducted through the atrioventricular node also belong to this category (fig 2).

Facilitation of unidirectional block and of slowing in conduction velocity within the reentry circuit are the most likely mechanisms for drug-induced worsening of an arrhythmia based upon reentry. By slowing intraventricular conduction class Ia and Ic drugs can

Table 1 Classification of the arrhythomogenic effects of antiarrhythmic drugs

(1) Worsening of an already manifest arrhythmia

(2) Induction of block or suppression of escape mechanism(s)

(3) Uncovering of a hidden arrhythmia mechanism

(4) Induction of a new arrhythmia mechanism

(5) Combinations of 1 to 4

Table 2 Worsening of an already manifest arrhythmia

Mechanism

- Creation of a more stable reentry circuit by shortening of the wavelength and/or widening of the echo zone.

- Decrease in atrial rate of atrial flutter or fibrillation leading to increased impulse conduction through the atrioventricular node.

Type of drugs Ia, Ic, II, IV, digitalis

Examples

- Extrasytoles $\rightarrow$ nonsustained tachycardia

Non-sustained tachycardia $\rightarrow$ sustained tachycardia

- Paroxysmal tachycardia $\rightarrow$ incessant tachycardia

- Slowing in atrial flutter rate $\rightarrow 1$ to 1 atrioventricula conduction familiar with the risk of drug treatment and to be able to recognise it. We suggest a practical approach to the different types of arrhythmogenesis that may develop after antiarrhythmic drug administration and we briefly review the possible types and mechanisms of arrhythmogenesis, which have been discussed extensively elsewhere. ${ }^{510-13}$ We also discuss the timing of the appearance of arrhythmogenesis after start of antiarrhythmic drug treatment, discuss the value of different non-invasive and invasive tests to document arrhythmogenesis or to recognise patients at an increased risk of

\footnotetext{
Maastricht, The Netherlands

Accepted for publication

1 November 1991

A Gorgels
} 
Figure 1 (A)

Ventricular extrasystoles in a patient with an old anteroseptal infarction.

(B) After treatment with the procainamide he developed sustained uniform ventricular tachycardia with the same $Q R S$ confirmation as the isolated ventricular

extrasystoles. In $A$ and $B$ the 12 electrocardiographic leads were recorded simultaneously.

Figure 2 Example of acceleration in ventricular rate after procainamide administration in a patien with atrial flutter.

(A) Atrial flutter (flutter interval 200 ms) with 2 to 1 atrioventricular relation resulting in a ventricular rate of $150 / \mathrm{min}$. (B)

During procainamide administration slowing in flutter rate (flutter interval 260/ms was followed by 1 to 1 atrioventricular conduction and $a$ ventricular rate of $230 / \mathrm{ms}$.
B

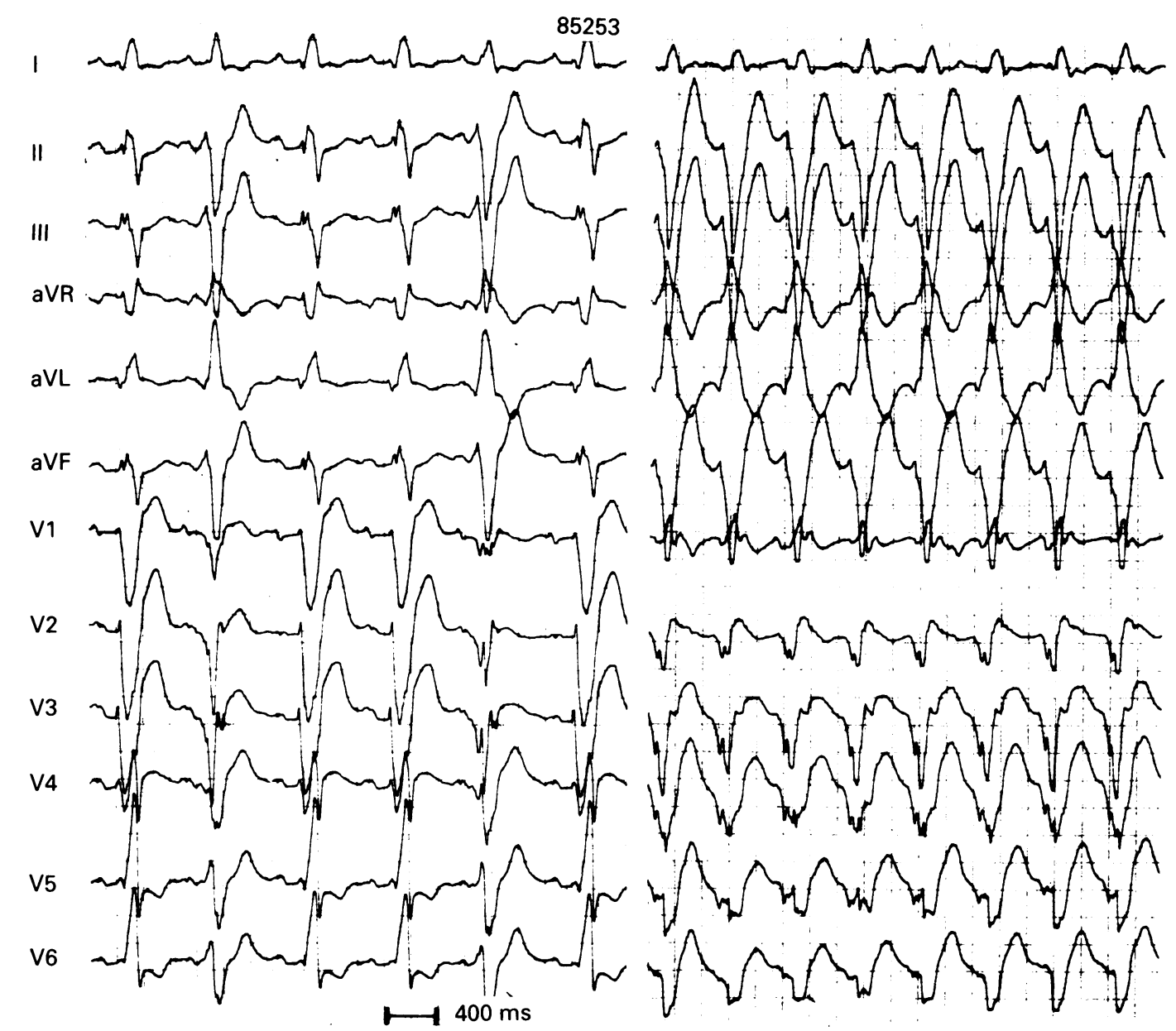

$90238 / 1$

Anqmantrat

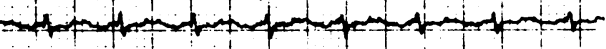

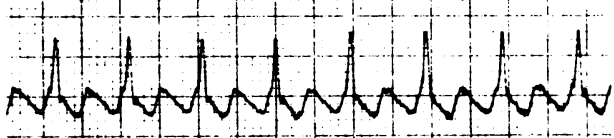

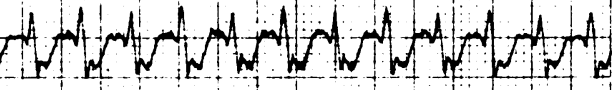

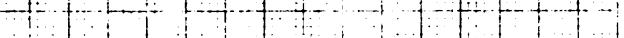

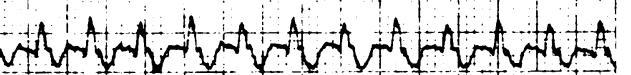
dubldowdult FLUTTER INTERVAL: $200 \mathrm{~ms}$

(1)

V3

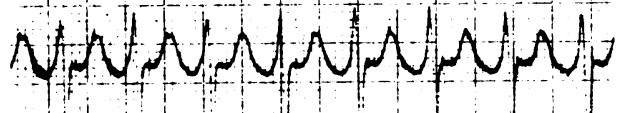

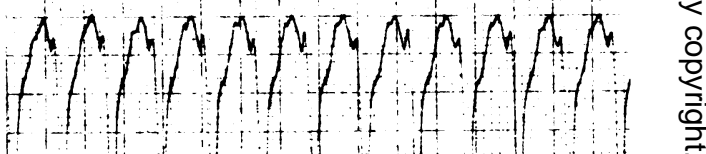

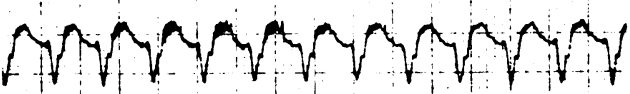

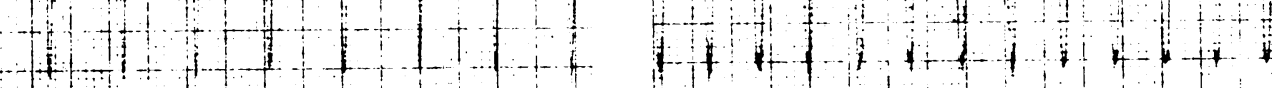

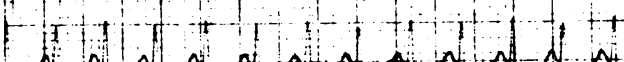
- N $f^{N} f^{N} f^{N} f^{N}$ 
A

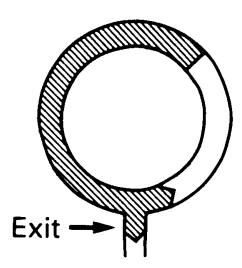

$W L=C V \times R P: \quad 10 \mathrm{~cm} / \mathrm{s} \times 250 \mathrm{~ms}=2.5 \mathrm{~cm}$

Circuit length:

$4 \mathrm{~cm}$

$150 / \mathrm{min}$

Figure 3 Possible mechanism of acceleration of tachycardia rate by a drug that slows conduction velocity but does not prolong the refractory period. $(A) A$ reentrant circuit with a length of $4 \mathrm{~cm}$ and a single exit point. The wavelength (WL) of the circulating impulse (the product of conduction velocity $(C V)$ and the refractory period $(R P)$ measures $10 \mathrm{~cm} / \mathrm{s} \times 250 \mathrm{~ms}=2.5 \mathrm{~cm}$. Each minute 150 impulses will leave the exit point. (B) After administration of a drug (such as a class Ic drug) that slows conduction without lengthening the refractory period $(R P)$ within the circuit. The wave-length now measures $7.5 \mathrm{~cm} / \mathrm{s} \times 250 \mathrm{~ms}=1.88 \mathrm{~cm}$. This means that two consecutive impulses can fit within the circuit of $4 \mathrm{~cm}$. Each minute $2 \times 113=226$ impulses will reach the exit point of the circuit. This mechanism of tachycardia acceleration after antiarrhythmic drug administration accords with the results of a recent study. ${ }^{14}$ facilitate the induction and perpetuation of ventricular arrhythmias. Paradoxically, drugs that slow intraventricular conduction may increase the rate of a tachycardia by using a smaller reentry circuit or by allowing two consecutive impulses within one reentry circuit $^{14}$ (figs 3-5). Drugs slowing intra-atrioventricular nodal conduction such as calcium antagonists, $\beta$ blocking agents, and digitalis may favour the continuation of a reentrant arrhythmia if the atrioventricular node is incorporated in the circuit.

\section{INDUCTION OF A BLOCK OR SUPPRESSION OF ESCAPE MECHANISMS}

Although the antiarrhythmic drug terminates or suppresses the ectopic rhythm it may worsen the condition of the patient because it also blocks conduction or suppresses escape mechanisms. Drugs can induce bradycardia and even asystole by creating sinus arrest or sinoatrial or atrioventricular block (table 3). Class Ib drugs may suppress atrioventricular junctional or ventricular escape mechanisms.
A

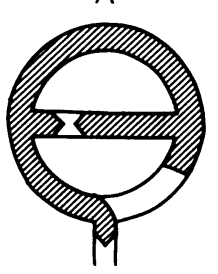

B

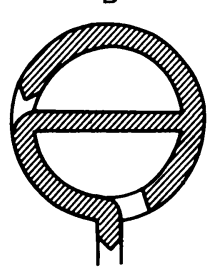

$W L=C V \times R P: \quad 10 \mathrm{~cm} / \mathrm{s} \times 250 \mathrm{~ms}=2.5 \mathrm{~cm} \quad 7.5 \mathrm{~cm} / \mathrm{s} \times 250 \mathrm{~ms}=1.88 \mathrm{~cm}$ Circuit length: $4 \mathrm{~cm}$

Exit activated:

$150 / \mathrm{min}$

$2 \mathrm{~cm}$

$225 / \mathrm{min}$

Figure 4 Another possible mechanism of tachycardia acceleration after administration of a drug that slows conduction velocity. Slowing of the conduction velocity means that a smaller reentry circuit can be used and this leads to more impulses leaving the exit point. See legend to fig 3 for abbreviation.
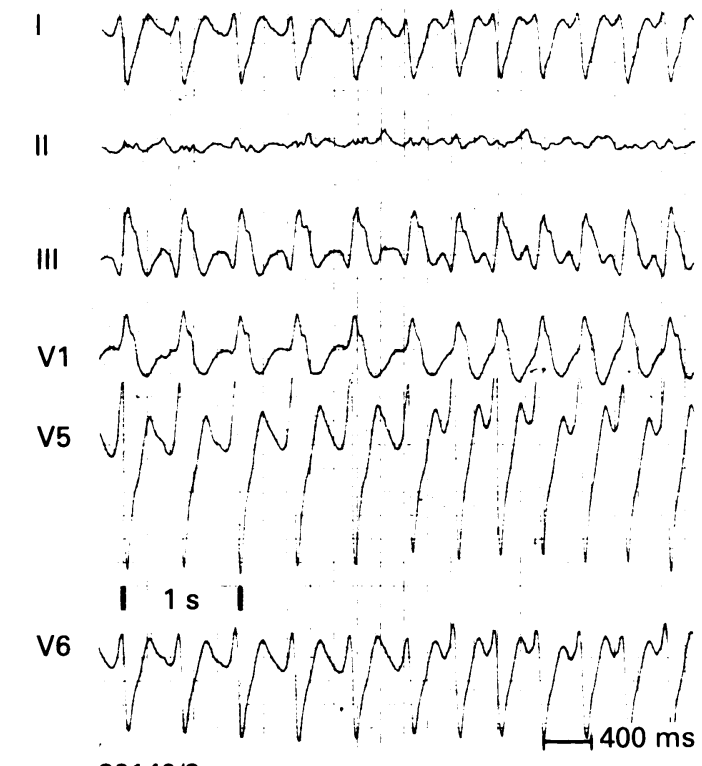

$82140 / 3$

Figure 5 Clinical example of sudden acceleration in rate of a ventricular tachycardia without change in $Q R S$ configuration during the intravenous injection of a class Ic antiarrhythmic drug. Possible mechanisms for this type of ventricular tachycardia acceleration include those shown schematically in figs 3 and 4.

\section{UNCOVERING A “HIDDEN" ARRHYTHMIA} MECHANISM

This category (table 4) includes patients in whom a dormant reentry circuit becomes manifest when an antiarrhythmic agent changes the refractory period and conduction velocity-for example, when a patient with an old myocardial infarction and ventricular extrasystoles develops sustained ventricular tachycardia after the administration of an antiarrhythmic drug (fig 6). This phenomenon can be seen after class Ia and class Ic drugs. These drugs slow myocardial conduction without prolonging the duration of the refractory period. This shortens the wave-length of the circulating impulse allowing it to "fit" within a reentry circuit.

INDUCTION OF A NEW ARRHYTHMIA MECHANISM An antiarrhythmic drug can induce a new

Table 3 Possible site( $s$ ) of block in relation to different types of antiarrhythmic agents

\begin{tabular}{llll}
\hline & \multicolumn{2}{l}{ Site of block } & \\
\cline { 2 - 3 } Class & Sinoatrial & Av nodal & His-Purkinje \\
\hline Ia & + & - & + \\
Ib & - & - & + \\
Ic & + & - & + \\
II & + & + & - \\
III & + & + & + \\
IV & - & + & - \\
Digitalis & + & + & - \\
\hline
\end{tabular}

Class Ia includes quinidine, procainamide, disopyramide, ajmaline; class Ib lignocaine, tocainide, mexiletine, dephenylhydantoin; class Ic encainide, flecainide, propafenone; Class II $\beta$ blocking agents; class III sotalol, amiodarone; clas $I V$ verapamil, diltiazem. 
Table 4 Uncovering of a hidden arrhythmia mechanism

Mechanism: Creation of a reentry circuit by causing unidirectional block and sufficient slowing in impulse unidirection

Type of drugs: Ia, Ic, III

arrhythmic mechanism (table 5)-for example the induction of early afterdepolarisations by class Ia and class III (sotalol, amiodarone) drugs leading to multiform ventricular tachycardia ${ }^{11-1315}$ (figs 7 and 8) or the induction of ectopic impulse formation based on later afterdepolarisations as in digitalis intoxication. ${ }^{16}$

\section{COMBINATIONS OF DIFFERENT MECHANISMS}

Arrhythmogenic effects after drug administration may also occur because of combinations of different mechanisms. Figure 9 shows that administration of a class Ic agent in a patient with the Wolff-Parkinson-White syndrome prevented atrial fibrillation but also allowed the development of incessant circus movement tachycardia, an arrhythmia that had not been clinically present before. In that patient the antiarrhythmic drug prevented atrial fibrillation from occurring and created complete anterograde block in the accessory pathway. Because of persistence of retrograde conduction through the accessory pathway circumstances were created for a circus movement tachycardia with atrioventricular conduction over the atrioventricular node and ventriculoatrial conduction over the accessory pathway. Another example is the patient with atrial fibrillation or atrial flutter who after treatment with a class Ic agent develops a high ventricular rate because a decrease in the number of atrial impulses allows more impulses to pass through the atrioventricular node to the ventricle. This increase in ventricular rate may result in rate dependent slowing in intraventricular conduction (manifest as widening of the QRS complex)

Figure 6 Example of the emergence of sustained uniform ventricular tachycardia after treatment of isolated ventricular extrasystoles (A) with a class Ic agent. Unlike fig 1 the $Q R S$ configuration of the ventricular extrasystole is completely different from the one seen during ventricular tachycardia. This suggests that $a$ "dormant" reentry circuit has been exposed by the administration of the antiarrhythmic agent.

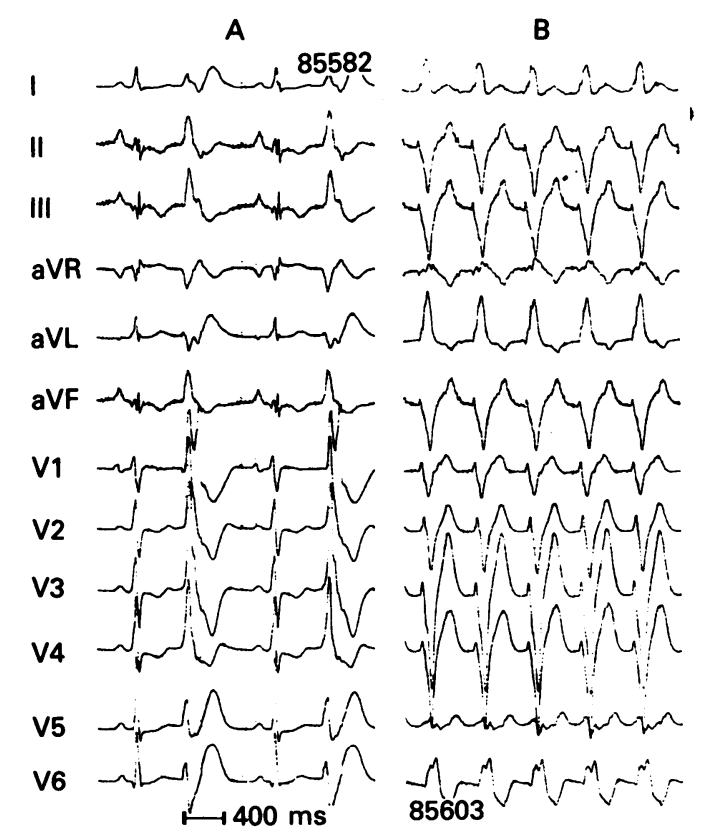

and the initiation of serious ventricular arrhythmias. ${ }^{17}$ This category includes patients in whom the administration of a class Ia or class III drug leads to the development of bradycardia (for example because of sinoatrial block) and facilitates the emergence of arrhythmias based uponoearly afterdepolarisations (torsades de pointes).

\section{Time of appearance of arrhythmogenic effects}

The onset of an arrhythmogenic effect after antiarrhythmic drug treatment can vary. Some proarrhythmic effects such as the occurrence of torsades de pointes after class Ia or class III drugs frequently occur early..$^{18}$ They are not dose related but based upon an idiosyncratic response to the drug. One dose of quinidine can be sufficient to initiate torsades de pointes ${ }^{19}$ (figs 7 and 8). However, as Nguyen et al pointed out ${ }^{20}$ half of the patients developing arrhythmogenic effects after class Ia drugs do so more than a week after the start of treatment. Arrhythmogenic effects can appear at so-called therapeutic drug concentrations not only because of a drug-induced shortening in wavelength of a circulating impulse resulting in a more stable reentry circuit, but also because of changes in electrolyte concentrations or because of ischaemia in the arrhythmogenic area. Arrhythmogenic effects can also occur when an antiarrhythmic drug reaches a toxic concentration as for example in digitalis intoxication.

Different types of arrhythmogenic effects can be caused by the same drug at different times after the start of its administration. Absence of one type of arrhythmogenesis does not mean that another type of arrhythmogenesis will not occur. A patient who does not develop torsades de pointes after the first dose of quinidine may show a change from a non-sustained to incessant ventricular tachycardia after two weeks of quinidine treatment when a dose favouring a more stable circulation of the impulse within a reentry circuit is reached. The CAST study showed increased arrhythmic events after encainide/flecainide during the whole period of administration of the antiarrhythmic drug. ${ }^{89}$ Table 6 suggests possible mechanisms for that finding.

Tests to recognise or document increased risk for arrhythmogenesis RISK PROFILE FOR THE DEVELOPMENT OF ARRHYTHMOGENIC EVENTS

It has repeatedly been stressed that the

Table 5 Induction of a new arrhythmia mechanism

Mechanism

- Early afterdepolarisation (EAD) leading to torsades de pointes

pointes

impulse formation in atrium, atrioventricular junction, and ventricle.

Type of drugs

- EAD: Ia, III

- DAD: digitalis 
Figure $7 \quad A$ simultaneously recorded 12 lead electrocardiogram of a patient with multiform The patient, a 24 year old woman had no symptoms and no structural cardiac abnormalities on echocardiography. ventricular extrasystoles.

aVR

aVL

aVF
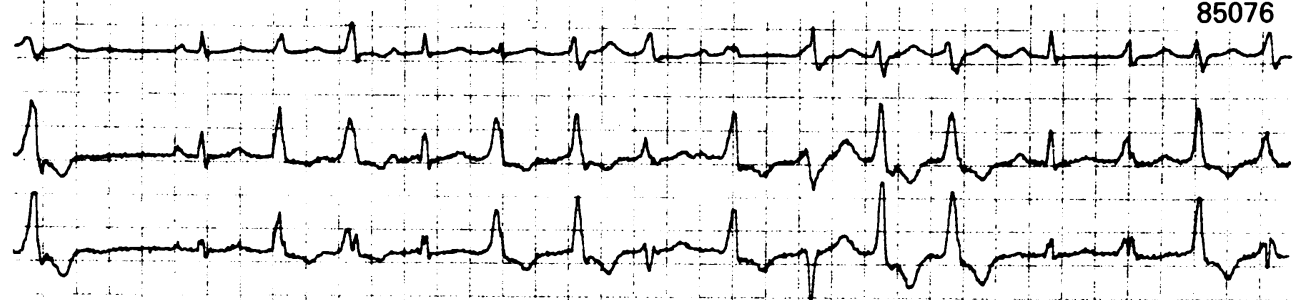

R
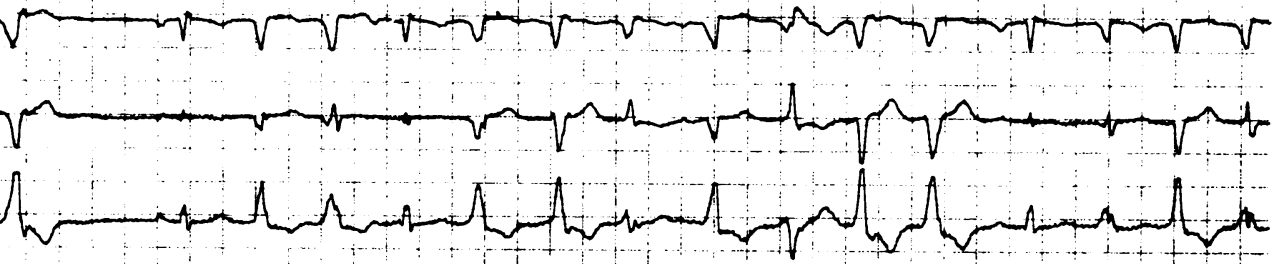

V1

V2

V3

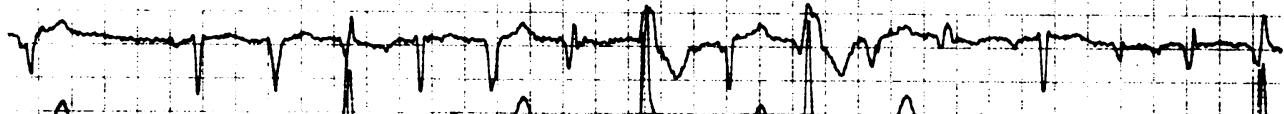

V4

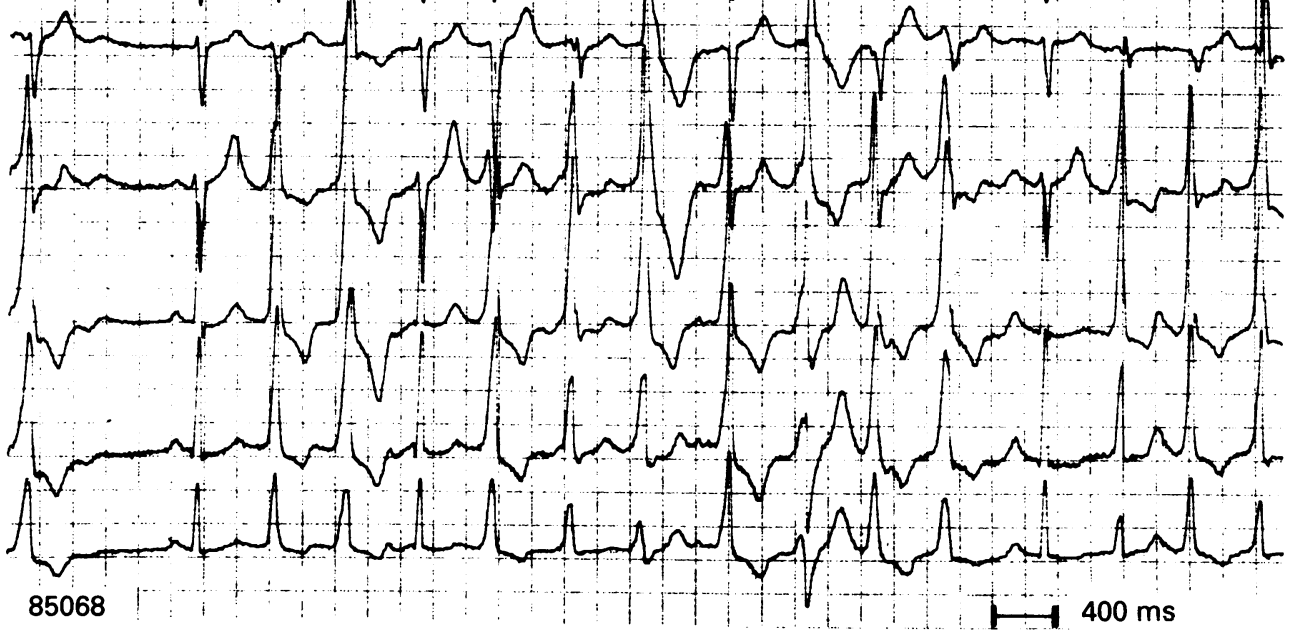

occurrence of an arrhythmogenic effect of an antiarrhythmic drug is unpredictable in individual patients. There are certain characteristics, however, that predispose patients to serious arrhythmogenic events or death during antiarrhythmic treatment. Morgenroth summarised these factors. ${ }^{22}$ They include severe structural heart disease, sustained ventricular tachycardia, in-hospital initiation of antiarrhythmic drug treatment, and large doses of antiarrhythmic drugs. Not surprisingly, the sicker the patient the higher the chance of an arrhythmia becoming worse after the start of antiarrhythmic drug treatment.

\section{TWENTY FOUR HOUR ELECTROCARDIOGRAPHIC RECORDING}

An arrhythmogenic effect may become manifest

Figure 8

Electrocardiogram from same patient as fig 7 after $200 \mathrm{mg}$ of quinidine sulphate. Note $Q T$ prolongation and the emergence of a torsades de pointes type of ventricular tachycardia. shortly after the start of treatment with an antiarrhythmic drug. Examples are the torsades de pointes type of multiform ventricular tachycardia after class Ia or class III drugs and the emergence of sustained uniform ventricular tachycardia in the patient with isolated ventricular extrasystoles or non-sustained ventricular tachycardia after the administration of class Ic drugs.

These changes can be documented by longterm electrocardiographic recording in the first days after the start of antiarrhythmic drug treatment, but as discussed previously, arrhythmogenic changes may also occur even later, so there are practical problems in using long-term electrocardiographic recording to recognise arrhythmogenic effects.

\section{EXERCISE TESTING}

Velebit et al emphasised the importance of exercise testing in evaluating the possible arrhythmogenic effects of antiarrhythmic agents. ${ }^{3}$ Antiarrhythmic drugs can induce rate related slowing of intramyocardial conduction (so-called use-dependent slowing). This can cause widening of the QRS complex with increasing heart rate. Recently, Ranger et al showed that some patients treated with flecainide develop considerable widening of their QRS complex followed by the emergence of ventricular tachycardia when they increase their heart rate by exercise. ${ }^{23}$ Figure 10 gives an

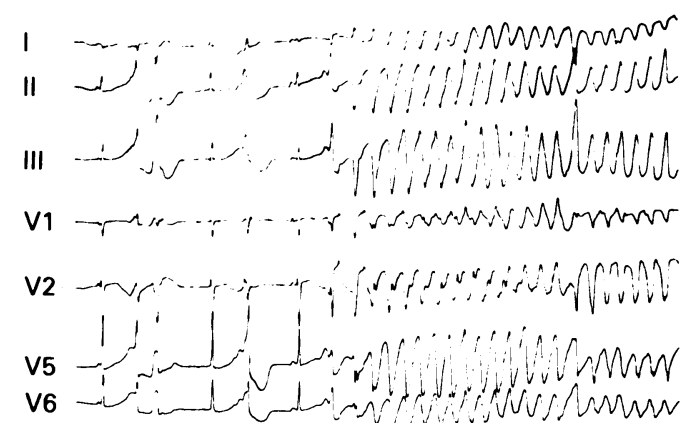

$400 \mathrm{~ms}$ 
Figure 9 (A) Atrial fibrillation in a patient with a left free wall accessory pathway. This patient had paroxysmal episodes of atrial fibrillation.

Administration of a class Ic drug produced anterograde block in the accessory pathway thereby protecting the patient against high ventricular rates during atrial fibrillation. The antiarrhythmic drug did not affect retrograde conduction over the accessory pathway, however, and the patient developed incessant circus movement tachycardia with atrioventricular conduction over the atrioventricular node and ventriculoatrial conduction over the accessory pathway.
A

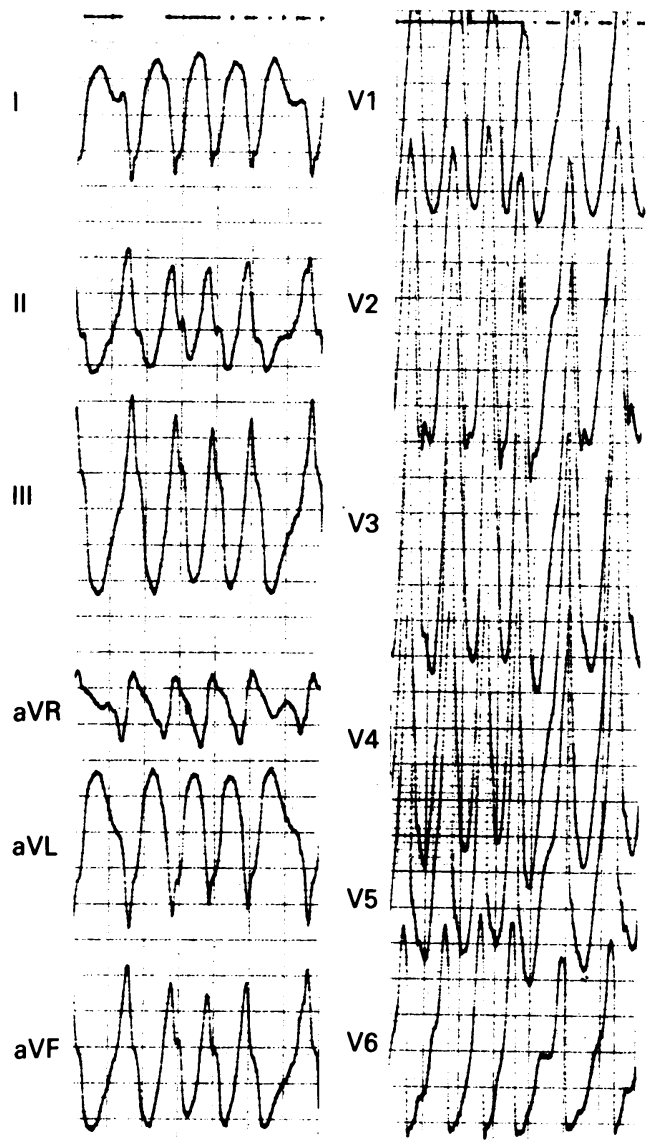

example of this phenomenon in a patient who was treated with a class Ic agent because of a non-sustained ventricular tachycardia. During administration of the antiarrhythmic drug the patient noticed that a sustained tachycardia always developed when he exercised. Figure 10 shows that during exercise testing the QRS complex and axis deviation increased considerably and was followed by sustained ventricular tachycardia. Exercise related ventricular tachycardia no longer occurred in this patient when the class Ic agent was replaced by a class III drug.

During exercise testing off drugs Kadish $e t$ $\mathrm{al}^{24}$ found a paradoxical increase in the corrected QT interval in patients who had arrhythmogenic effects on a type Ia drug. Antiarrhythmic drugs may not only produce use-dependent slowing in conduction but also use-dependent prolongation of the refractory period. Figures 11 and 12 show that in a patient with right bundle branch block and left anterior hemiblock a rate-related (use-depen-

Table 6 Possible mechanisms of deleterious effects of class Ic agents after myocardial infarction

(a) Negative inotropic effects.

(b) Rate related slowing of intramyocardial conduction favouring initiation of ventricular tachycardia on exercis or after onset of atrial flutter or atrial fibrillation.

(c) Slowing in intramyocardial conduction during ischaemi favouring initiation of ventricular tachycardia. ${ }^{2}$

(d) Induction of slow heart rates because of sinoatrial or subnodal block.

(e) Acceleration of ventricular tachycardia because slowing in intramyocardial conduction may allow two circulating impulses within one reentry circuit or the use of a smalle reentry circuit. dent) block was seen in the left posterior fascicle after flecainide administration. The observations of Ranger $e t a l^{23}$ and Kadish and coworkers ${ }^{24}$ indicate the need for prospective studies before and during antiarrhythmic drug administration to establish the role of exercise testing in the risk estimation of arrhythmogenesis.

\section{PROGRAMMED STIMULATION OF THE HEART}

Several groups of investigators reported on the use of programmed stimulation of the heart to identify the arrhythmogenic effects of antiarrhythmic drugs. ${ }^{25-28}$ Induction of a sustained tachycardia in a patient in whom before drug administration only a non-sustained tachycardia could be induced, the initiation of a faster tachycardia by fewer premature beats, and the emergence of incessant tachycardia after antiarrhythmic drug administration have all been regarded as signs of the arrhythmogenic effects of antiarrhythmic agents. The lack of information on the effect of long-term oral drug administration in patients showing the arrhythmogenic "effects" of that drug during programmned electrical stimulation, made us reluctant to regard the development of the arrhythmogenic effects during programmed stimulation as a contraindication to the chronic oral use of such drugs. Also because Ross et al reported considerable variation in the number of premature stimuli required to induce sustained ventricular tachycardia on different days $^{29}$ we decided to continue antiarrhythmic drug treatment irrespective of the findings 
Figure 10 Initiation of sustained ventricular tachycardia during

exercise testing in a patient treated with class Ic agent. (A) Electrocardiogram before exercise. The heart rate was 80 beats/min and the $Q R S$ width measured $100 \mathrm{~ms}$. (B) During exercise the heart rate was 130 beats/min with a $Q R S$ width of $160 \mathrm{~ms}$ and left axis deviation. Shortly thereafter (C) a ventricular tachycardia started with a rate of 150 beats/min.
A

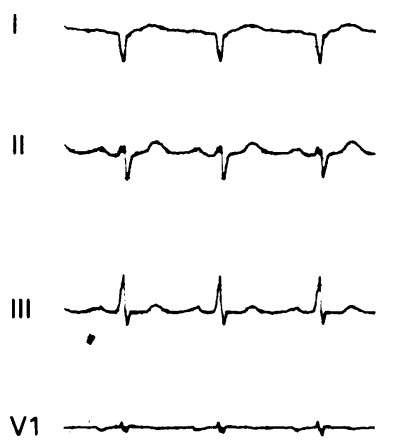

V4<smiles>CCC1CCC(CCC2CCCC2)C1</smiles>

V6

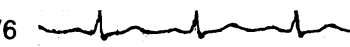

B

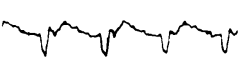<smiles>C=CCC(C)CCC(C)CCCCCC</smiles>

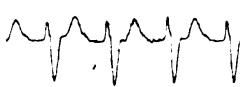

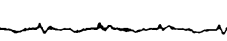

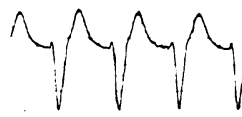<smiles>CCCCCCCCCCCCCCCCCCCC</smiles>

obtained during the programmed stimulation study. No attention was given to the induction of a sustained ventricular tachycardia during drug treatment, when before the drug only a non-sustained arrhythmia could be initiated. The drug was also continued despite induction of a faster tachycardia or induction by fewer premature stimuli. During follow up there were no differences in the occurrence of spontaneous sustained ventricular arrhythmias or of sudden death between patients with or without easier induction or change from induction of a non-sustained to a sustained ventricular tachycardia after antiarrhythmic drug administration. ${ }^{30}$ We think that only the induction of an incessant tachycardia after antiarrhythmic drug administration can be accepted as definite evidence of an arrhythmogenic effect of the drug.

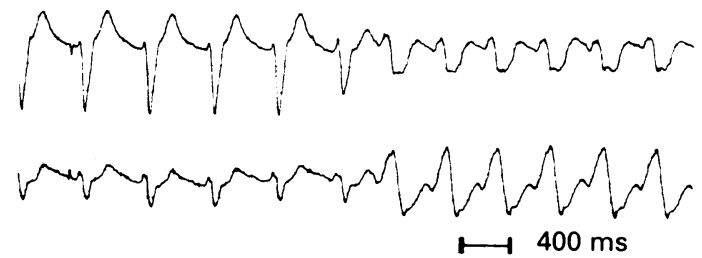

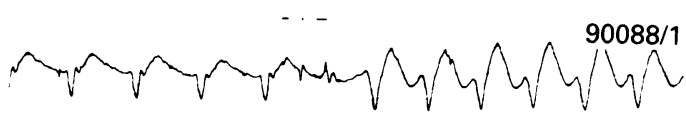

s.

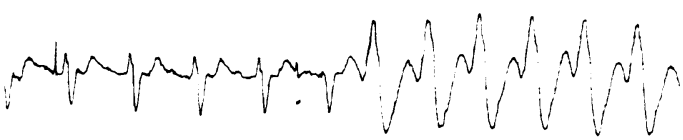

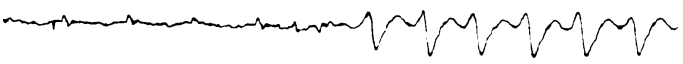

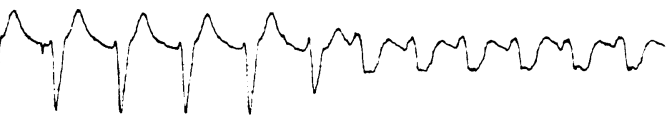

\section{The practical approach}

Antiarrhythmic drug treatment should be started only when it is clearly indicated by symptoms and/or an arrhythmia with a bad prognosis. ${ }^{31}$ The rules outlined in table 7 should be followed to minimise the arrhythmogenic effects and to aid their early recognition. In view of the considerable pharmacokinetic and pharmacodynamic differences between antiarrhythmic drugs we believe that physicians treating cardiac antiarrhythmias should familiarise themselves with selected antiarrhythmic agents and known how these drugs are metabolised and excreted and how they are affected by other medication given concomitantly. In patients with structural heart disease antiarrhythmic drug treatment should be started in hospital, preferably under continuous electrocardiographic monitoring. Exercise testing should be
Figure 11 Example of use-dependent prolongation of the refractory period. The recording is from a patient with paroxysmal atrial tachycardia who developed episodes of syncope after being treated with flecainide. Note that atrioventricular conduction goes over the left posterior fascicle. During flecainide administration complete atrioventricular block occurred shortly after a few beats of atrial tachycardia. The trace was made after a pacing wire was inserted. Asystole was terminated by a paced ventricular complex.

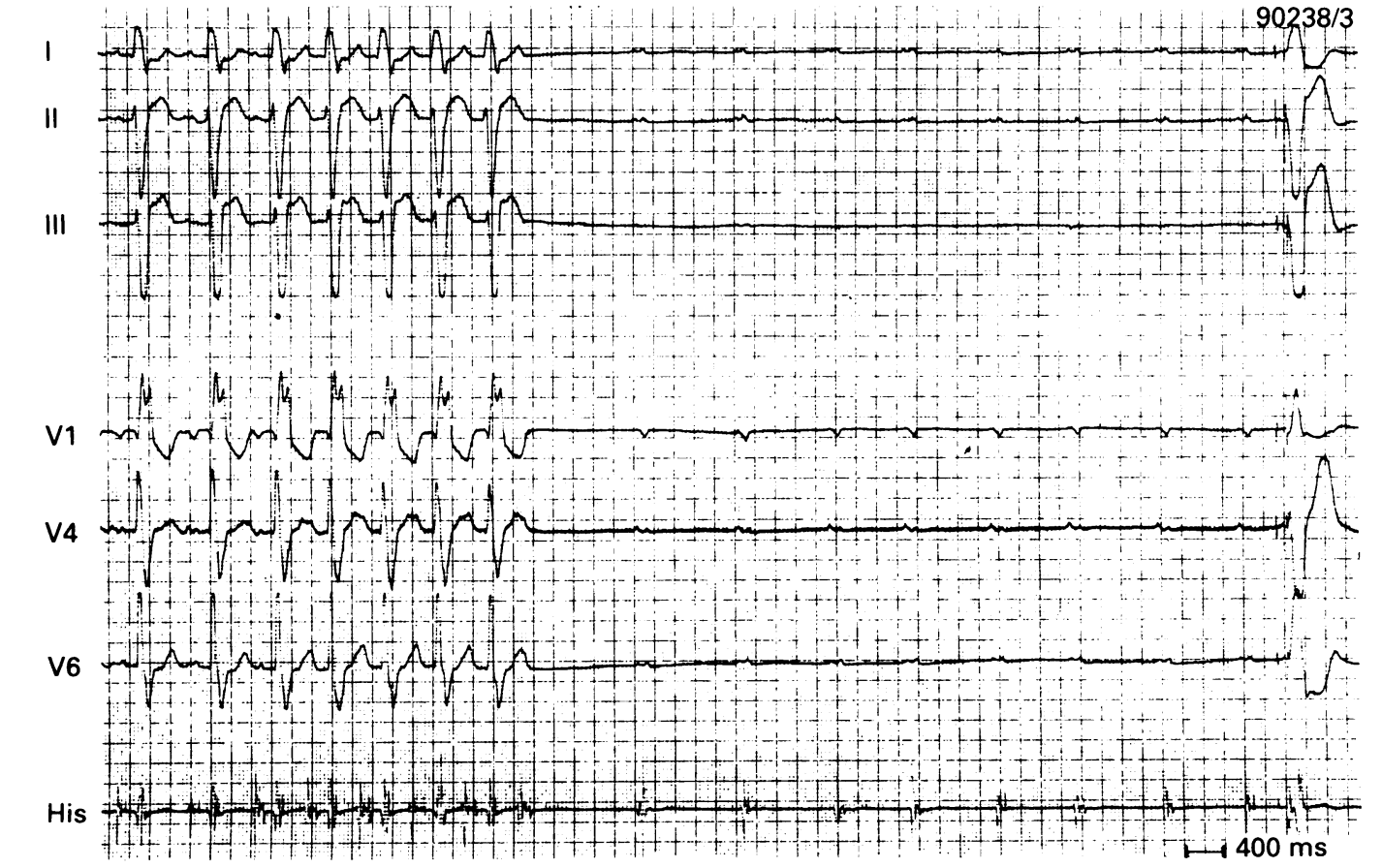


Figure 12

Electrocardiogram from the same patient as figure 11 after flecainide treatment was stopped. Note that rate acceleration during atrial tachycardia is no longer followed by complete atrioventricular block. Figures 11 and 12 suggest that flecainide induced rate-related (usedependent) prolongation of the refractory period of the left posterior fascicle was responsible for complete atrioventricular block.

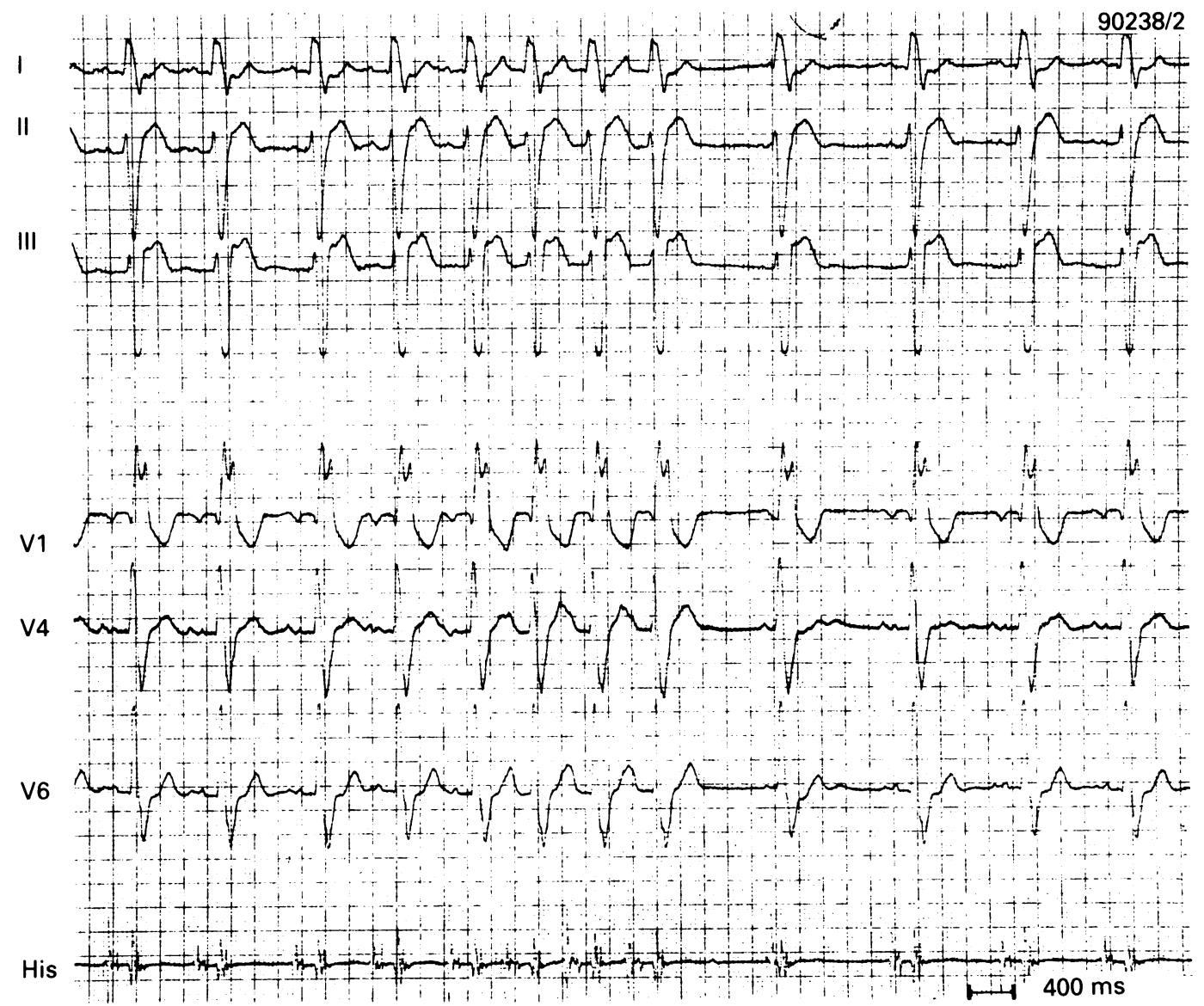

performed before and during antiarrhythmic drug administration. The patient should be instructed about the signs and symptoms of worsening of the existing arrhythmia or the appearance of a new one.

\section{Conclusions}

Antiarrhythmic agents commonly, have arrhythmogenic effects that are life-threatening and these can occur as long as the patient is taking antiarrhythmic medication. Although, as outlined, certain measures can be taken to minimise the chance of arrhythmogenic effects, the patient and the physician should constantly be alert to this possibility.

Table 7 Measures to minimise arrhythmogenic effects of antiarrhythmic drugs

(a) Be certain that the patient needs antiarrhythmic drug treatment.

(b) Identify and correct cardiac ischaemia and pump function abnormalities.

(c) Correct electrolyte abnormalities.

(d) Use an antiarrhythmic drug you know well.

(e) Start treatment in hospital while monitoring the ECG if the patient has structural heart disease.

(f) Perform exercise testing before and during drug administration.

(g) Instruct the patients about signs and symptoms of worsening of existing or appearance of new arrhythmias.

h) Regularly check the function of the organs responsible for breakdown and excretion of the antiarrhythmic drug.

(i) Know the possible effects of additional medication on the concentration of the antiarrhythmic drug.
1 Krikler DM. A fresh look at cardiac arrhythmias. Lancet 1974;1:913-7.

2 Krikler DM, Curry PVL. Torsade de pointes, an atypical ventricular tachycardia. Br Heart J 1976;38:117-20.

3 Velebit V, Podrid P, Lown B, Cohen BH, Graboys TB. Aggravation and provocation of ventricular arrhythmia by antiarrhythmic drugs. Circulation 1982;65:886-94.

4 Ruskin JN, McGovern MB, Garan H, DiMarco JP, Kelly E. Antiarrhythmic drugs: a possible cause of out-of-hospital cardiac arrest. N Engl J Med 1983;309:1302-6.

5 Rosen MR, Wit AL. Arrhythmogenic actions of antiarrhythmic drugs. Am J Cardiol 1987;59:10E-8E.

6 Bigger JT, Sahar DJ. Clinical types of proarrhythmic response to antiarrhythmic drugs. $A m J$ Cardio 1987;59:2E-9E.

7 Brugada P, Wellens HJJ. Arrhythmogenesis of antiarrhythmic drugs. Am J Cardiol 1988;61:1108-11.

8 The Cardiac Arrhythmia Suppression Trial (CAST) Investigators. Preliminary report: effect of encainide and flecainide on mortality in a randomised trial of arrhythmia suppression after myocardial infarction. $N \mathrm{Engl} \mathrm{J} \mathrm{Med}$ 1989;321:406-12.

9 Echt DS, et al. Mortality and morbidity of patients receiving encainide flecainide or placebo. The Cardiac Arrhythmia encainide flecainide or placebo. The Cardiac Arrh

10 Woosley RL, Roden DM. Pharmacologic causes of arrhythmogenic actions of antiarrhythmic drugs. Am J Cardiol mogenic actions of

11 Cranefield PD, Aronson RS. Cardiac arrhythmias: the role of triggered activity and other mechanisms. Mount Kisco: Futura, 1988.

2 Zipes DP. Cardiac electrophysiology: promises and contributions. J Am Coll Cardiol 1989;13:1329-52.

13 Jackman WM, Szabo B, Friday KJ, et al. Ventricular tachyarrhythmias related to early afterdepolarisations and triggered firing: relationship to QT interval prolongation and potential therapeutic role for calcium channel block-

14 Brugada J, Boersma L, Kirchoff C, et al. Double-wave reentry as a mechanism of acceleration of ventricular tachycardia. Circulation 1990;81:1633-43.

15 Jackman WM, Friday KJ, Clark M, et al. The long QT syndromes: A critical review, new clinical observations and a unifying hypothesis. Prog Cardiovasc Dis and a unifying

16 Gorgels APM, Beekman HDM, Brugada P, Dassen WRM, Richards DAB, Wellens HJJ. Extrastimulus-related shoring agents. J Cardiovasc Electrophysiology 1990;1:170-95. 
tening of the first post-pacing interval in digitalis-induced ventricular tachycardia: observations during programmed electrical stimulation in the conscious dog. $\mathrm{J} \mathrm{Am} \mathrm{Coll}$ Cardiol 1983;1:840-57.

17 Crijns HJ, Van Gelderen JC, Lie KI. Supraventricular tachycardia mimicking ventricular tachcycardia during tachycardia mimicking ventricular tachcycardia dur

18 Minardo JD, Heger JJ, Miles WM, Zipes DP, Prystowsky EN. Clinical characteristics of patients with ventricular Med 1988;319:257-62.

19 Roden DM, Woosley RL, Primm RK. Incidence and clinical features of the quinidine-associated long QT syndrome: implication for patient care. Am Heart $J$ 1986;111 1088-93.

20 Nguyen PT, Scheinman MM, Segar J. Polymorphic ventricular tachycardia: clinical characteristics, therapy and the QT interval. Circulation 1986;74:340-9.

21 Nattel S, Pedersen DH, Zipes DP. Alterations in regional myocardial distribution and arrhythmogenic effects of aprinidine produced by coronary artery occlusions in the dog. Cardiovasc Res 1981;15:80-5.

22 Morgenroth J. Risk factors for the development of proarrhythmic events. Am J Cardiol 1987;59:32E-7E.

23 Ranger S, Talajic M, Lemery R, Roy D, Nattel S. Amplification of flecainide-induced ventricular conduction slowing by exercise: a potentially significant clinical consequence of by exercise: a potentially significant clinical consequence of use-dependent

24 Kadish AH, Weisman HF, Veltri EP, Epstein AE, Slepian MJ, Levine JH. Paradoxical effects of exercise on the QT interval in patients with polymorphic ventricula tachycardia receiving type Ia antiarrhythmic agents. Circulation 1990;81:14-9.

25 Rinkenberger RL, Prystowsky EN, Jackman WM, Naccarelli GV, Heger JJ, Zipes DP. Drug conversion of nonsustained ventricular tachycardia to sustained ventricular tachycardia during serial electrophysiologic studies: Identification of drugs that exacerbate tachycardia studies: Identification of drugs that exacerbate tachycardia and potential mechanism. Am Heart J 1982;103:177-84. 26 Poser RF, Podrid PJ, Lombardi F, Lown B. Aggravation of arrhythmia induced with antiarrhythmic drugs durin

27 Au PK, Bhandari AK, Bream R, Schreck D, Siddiqi R Rahimtoola SH. Proarrhythmic effects of antiarrhythmic drugs during programmed ventricular stimulation in patients without ventricular tachycardia. J Am Coll Cardiol 1987;9:389-97.

28 Horowitz LN, Greenspan AM, Rae AP, Kay HR, Spielman SR. Proarrhythmic responses during electrophysiologic testing. Am J Cardiol 1987;59:45E-8E.

29 Ross DL, Cooper MJ, Ho B, et al Day-to-day reproducibility of ventricular tachycardia induction at electrophysiologic study. Circulation 1984;70:399-412.

30 Brugada $\mathbf{P}$, Lemery $R$, Talajic $M$, Della Bella $P$, Wellens HJJ Treatment of patients with ventricular tachycardia HJJ. Treatment of patients with ventricular tachycardia or ventricular fibrillation. In: Brugada P, Wellens HJJ, eds. Futura, 1987:457-70.

31 Wellens HJJ, Brugada $P$. Treatment of cardiac arrhythmias: when, how and where? J Am Coll Cardiol 1989;14: 1417-28. 